Portfoliomanagement in Unternehmen 
Reinhard Grimm • Markus Schuller Raimund Wilhelmer

\section{Portfoliomanagement in Unternehmen}

Leitfaden für Manager und Investoren

Springer Gabler 
Reinhard Grimm

St. Pölten

Österreich

Markus Schuller

Monaco

Monaco
Raimund Wilhelmer

Haslau/Wien

Österreich

Die Deutsche Nationalbibliothek verzeichnet diese Publikation in der Deutschen Nationalbibliografie; detaillierte bibliografische Daten sind im Internet über http://dnb.d-nb.de abrufbar.

\section{Springer Gabler}

(c) Springer Fachmedien Wiesbaden 2014

Das Werk einschließlich aller seiner Teile ist urheberrechtlich geschützt. Jede Verwertung, die nicht ausdrücklich vom Urheberrechtsgesetz zugelassen ist, bedarf der vorherigen Zustimmung des Verlags. Das gilt insbesondere für Vervielfältigungen, Bearbeitungen, Übersetzungen, Mikroverfilmungen und die Einspeicherung und Verarbeitung in elektronischen Systemen.

Die Wiedergabe von Gebrauchsnamen, Handelsnamen, Warenbezeichnungen usw. in diesem Werk berechtigt auch ohne besondere Kennzeichnung nicht zu der Annahme, dass solche Namen im Sinne der Warenzeichen- und Markenschutz-Gesetzgebung als frei zu betrachten wären und daher von jedermann benutzt werden dürften.

Lektorat: Stefanie Brich, Katharina Harsdorf

Gedruckt auf säurefreiem und chlorfrei gebleichtem Papier

Springer Gabler ist eine Marke von Springer DE. Springer DE ist Teil der Fachverlagsgruppe Springer Science+Business Media

www.springer-gabler.de 


\section{Vorwort}

Vor nicht allzu langer Zeit stand ein mittelständisches österreichisches Unternehmen mit etwa 1.000 Mitarbeitern vor der Herausforderung, dass die Umsätze in einem der adressierten Zielmärkte stark rückläufig waren. Daher wurde der Portfoliomanager mit der Aufgabe betraut, eine Marktanalyse durchzuführen, um die Positionierung der einzelnen Produkte anzupassen und potenzielle neue Marktsegmente zu identifizieren. Es verging Woche um Woche, ohne dass eine entsprechende Erhebung vorlag. Der zuständige Manager, der die Analyse in Auftrag gegeben hatte, wurde zusehends nervöser, da keine Gegenmaßnahme für den anhaltenden Rückgang definiert werden konnte, und übte zunehmend Druck auf den Portfoliomanager aus. Trotzdem hielt er auch nach einem Zeitraum von zwei Monaten das erwünschte Dokument nicht in Händen. Eines Tages sprach ihn ein Kollege des Portfoliomanagers auf dem Gang an und teilte ihm mit, dass der betroffene Mitarbeiter fast rund um die Uhr an der Analyse arbeite, aber sich sein Elaborat nicht zu übermitteln getraue, da er ja nicht wisse, ob das Ergebnis „richtig“ sei. In seiner perfektionistischen Art wollte der Portfoliomanager eine hochwertige Arbeit abliefern, hatte aber keinen Maßstab dafür, diese inhaltlich zu bewerten.

Diese und zahlreiche vergleichbare Begebenheiten, die wir in den letzten Jahren beobachten konnten, waren der Anstoß, ein Buch zum Thema Portfoliomanagement zu schreiben. Portfoliomanagement ist mit Sicherheit eine der ambivalentesten Aufgaben in einem Unternehmen. In keiner anderen Rolle ist das Spektrum der Anforderungen derart vielfältig und wohl wenige Funktionen sind so konfliktbehaftet wie das Portfoliomanagement. Dies fordert einen speziellen Typ Mensch, der eine breite Palette an fachlicher Qualifikation, aber auch an sozialen Fähigkeiten an den Tag legt. Allerdings sind am Arbeitsmarkt nur wenige solcher „Wunderwuzzis“ zu bezahlbaren Konditionen verfügbar und darüber hinaus wird auch der Wert der Tätigkeiten kraft ihrer indirekten Wirkung häufig deutlich unterschätzt. Daher kommt es in Unternehmen, die Produkt- oder Portfoliomanagement einführen, oftmals zu pragmatischen Besetzungen durch gute Fachkräfte, ehemalige Team- oder Projektleiter sowie Kollegen aus dem Marketing, die mit der Aufgabe betraut werden, das „Portfolio des Unternehmens zu konsolidieren und zu managen“. Die Konsequenz zeigt sich in obigem Beispiel. In diesem Fall wurde ein hervorragender, analytisch veranlagter Mitarbeiter vom Chefentwickler zum Portfoliomanager „befördert“. 
Dieser stand nun vor der Herausforderung, eine Arbeit durchzuführen, deren Richtigkeit erst Jahre später beurteilt werden kann und keinesfalls durch ihn selbst zum Zeitpunkt der Erstellung.

In diesem Buch wollen wir daher vor allem die unternehmerische Natur der Aufgabe des Portfoliomanagements in ihrer Gesamtheit verdeutlichen und darüber hinaus auch auf die Voraussetzungen eingehen, die für deren Gelingen unerlässlich sind. Für Entscheider und Unternehmer soll es Anhaltspunkte liefern, die Rahmenbedingungen für Portfoliomanagement richtig zu definieren, die optimale Person für diese Aufgabe auszuwählen und deren Arbeit zu bewerten. Umgekehrt möchten wir auch für Portfoliomanager mit konkreten Beispielen, Tipps und Handlungsanregungen eine Unterstützung für die vielfältigen Aspekte ihrer Rolle bieten - wissend, dass eine Person wohl nur schwer das gesamte Spektrum abdecken kann.

Dieser Umstand hat uns auch dazu bewogen, zu dritt an diesem Buch zu schreiben. Raimund Wilhelmer bringt als Unternehmer und Berater vor allem Erfahrungen im Aufbau kleiner und mittelständischer Firmen ein, die sich mit Produkten am Markt erfolgreich positionierten. Den Blickwinkel größerer, international tätiger Unternehmen trägt Reinhard Grimm bei. Er kennt aus seiner beruflichen Praxis auch die Herausforderungen, mit denen sich Portfoliomanager angesichts komplexer Organisationsstrukturen und interner Vorgaben auseinanderzusetzen haben. Von ihm stammen vor allem jene Aspekte, die es zu berücksichtigen gilt, um in einem solchen Kontext ein funktionierendes Portfoliomanagement aufzubauen und nachhaltig zu etablieren. Da es beim Management von Produktportfolios in den meisten Fällen letztlich auch darum geht, entsprechende Einnahmen und Gewinne zu erwirtschaften, betrachtet Markus Schuller das Thema Portfoliomanagement aus Investorensicht und steuert übertragbare Erkenntnisse aus der Optimierung von Portfolios im Asset Management bei. Er ist Eigentümer und Geschäftsführer eines Unternehmens, das sich auf die Optimierung der Strategic Asset Allocation von Portfolios professioneller Anleger in Europa fokussiert, und kennt die Kriterien, nach denen Portfolios und Geschäftspläne beurteilt werden.

Die Beispiele, die wir in diesem Buch anführen, basieren neben frei erfundenen Illustrationen vor allem auf öffentlich zugänglichen Publikationen und realen Situationen, die wir selbst in der Praxis miterleben durften - bei denen jedoch die betroffenen Personen und Unternehmen aus Gründen der Vertraulichkeit mit fiktiven Namen bezeichnet werden.

Wir hoffen, Ihnen damit einen vielschichtigen Einblick in das Thema Portfoliomanagement zu vermitteln und dass Sie für sich selbst zahlreiche nützliche Anregungen aus diesem Buch ableiten können.

In jedem Fall wünschen wir Ihnen viel Freude beim Lesen und vor allem Erfolg bei Ihren Vorhaben rund um das Thema Portfoliomanagement!

Wien, November 2013

Reinhard Grimm

Markus Schuller

Raimund Wilhelmer 


\section{Inhaltsverzeichnis}

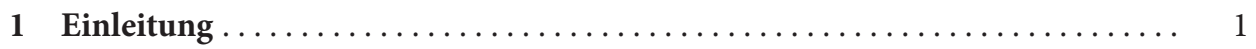

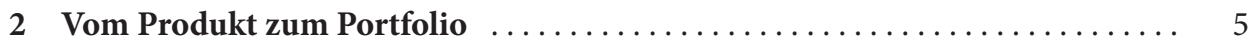

2.1 Produkt oder Einzellösung? .......................... 5

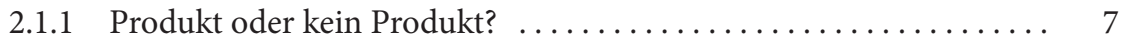

2.1.2 Typische Muster halbherziger Produktentscheidungen ........ 11

2.1.3 Die Falle der „kleinen Anpassungen“ .................. 14

2.1.4 Zwei Geschäftsmodelle . . . . . . . . . . . . . . . . . . . . . . . 16

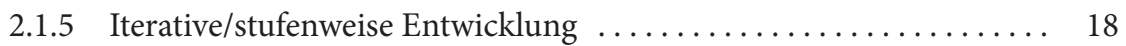

2.2 Mehrere Abstraktionsebenen von Produkten $\ldots \ldots \ldots \ldots \ldots \ldots \ldots \ldots . \ldots \ldots$

2.2.1 Ebene 1: Direkte Replikation des primären Erzeugnisses ....... 21

2.2.2 Ebene 2: Metaprodukt ......................... 23

2.2.3 Ebene 3: Vorgehensweise als Produkt $\ldots \ldots \ldots \ldots \ldots \ldots \ldots \ldots$

2.3 Ein einzelnes Produkt birgt Risiken - mehrere

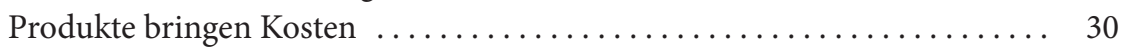

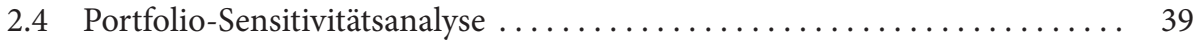

2.5 Fokussierung versus Diversifikation $\ldots \ldots \ldots \ldots \ldots \ldots \ldots \ldots \ldots \ldots \ldots$

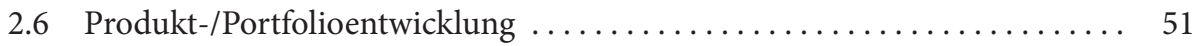

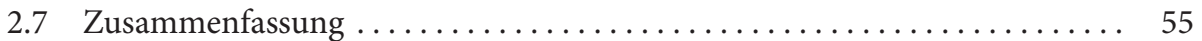

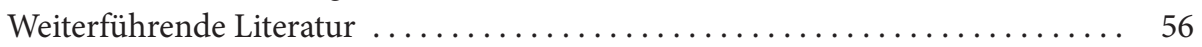

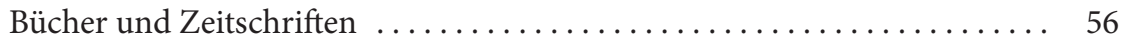

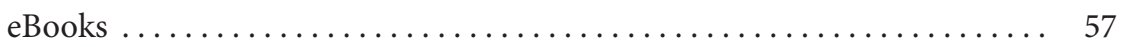

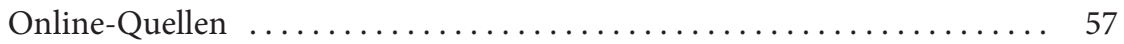

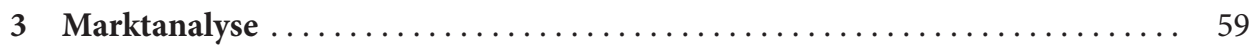

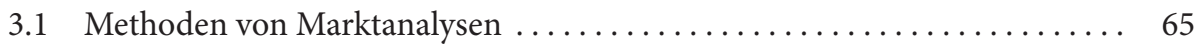

3.1.1 Abgrenzung der Analysevariablen .................. 67

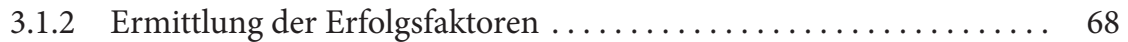

3.1.3 Auswahl der Datenquellen .................... 70

3.1.4 Verwendete Methoden der Marktanalyse .............. 73 


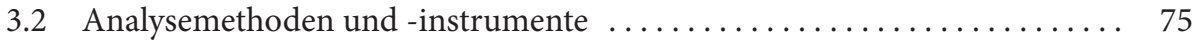

3.2.1 Portfolio-Markt-Matrix (Ansoff-Matrix oder Z-Matrix) . . . . . . . 75

3.2.2 Marktwachstums-Marktanteils-Portfolio (BCG-Matrix oder

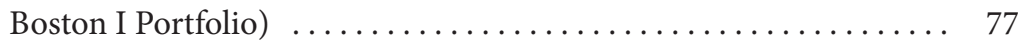

3.2.3 Marktattraktivitäts-Wettbewerbsstärken-Portfolio (Neun-Felder-Portfolio oder McKinsey-Portfolio) ......... 79

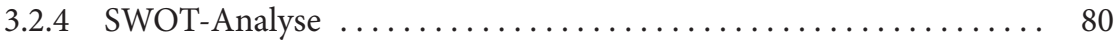

3.2.5 Aussagekraft von Marktanalysen $\ldots \ldots \ldots \ldots \ldots \ldots \ldots \ldots . \ldots . \ldots 1$

3.3 Wie ermittelt man Marktpotenzial und in weiterer

Folge potenzielles Absatzvolumen? ...................... 83

3.4 Regelmäßige Marktbeobachtung $\ldots \ldots \ldots \ldots \ldots \ldots \ldots \ldots \ldots \ldots \ldots \ldots . \ldots \ldots$

3.5 Marktanalyse im Kontext interner und externer Unternehmenskomplexität $\ldots \ldots \ldots \ldots \ldots \ldots \ldots \ldots \ldots \ldots . \quad 87$

3.5.1 Interne und externe Komplexität $\ldots \ldots \ldots \ldots \ldots \ldots \ldots \ldots \ldots . \ldots$

3.5.2 Erkennen und Verarbeitung von Komplexität ............. 91

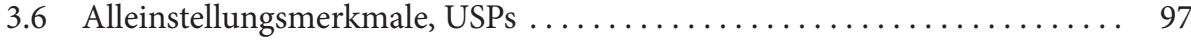

3.6.1 Merkmale von USPs . . . . . . . . . . . . . . . . . . . . 98

3.6.2 Individuelle Einschätzung versus analytisches Erkennen . . . . . . 99

3.7 Innovationskraft: „Early Bird“ versus „Adabei“ . . . . . . . . . . . . . . . . . . 100

3.7.1 Innovationen und deren Potenzial $\ldots \ldots \ldots \ldots \ldots \ldots \ldots \ldots \ldots$

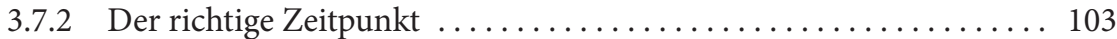

3.8 Kosten versus Nutzen von Marktanalysen $\ldots \ldots \ldots \ldots \ldots \ldots \ldots \ldots \ldots \ldots . \ldots \ldots$

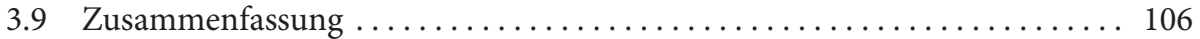

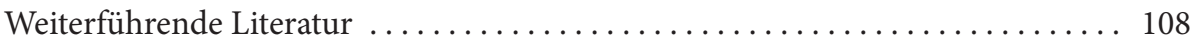

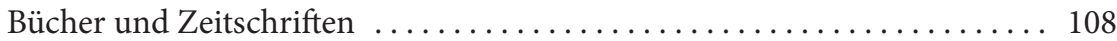

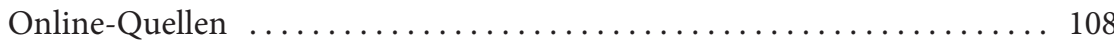

4 Das Produktportfolio im Kontext der Unternehmensstrategie . . . . . . . . . 111

4.1 Instrumente des strategischen Marketings - der Marketingprozess . . . . . 113

4.2 Wie erstellt man eine interne Unternehmensanalyse? . . . . . . . . . . . . 117

4.2.1 Kennzahlen-Analyse ......................... 117

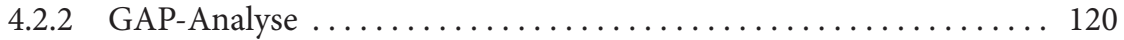

4.2.3 Wert(schöpfungs)ketten-Analyse ................. 123

4.3 Benchmark-Analyse als Schnittstelle zwischen

Markt und Unternehmen . . . . . . . . . . . . . . . . . . . . . . . . . . . . 125

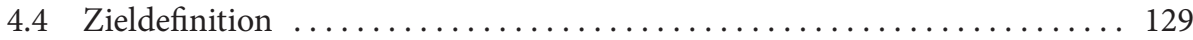

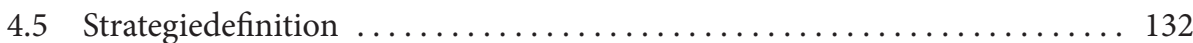

4.5.1 Definition des Portfolios: Portfoliostrategie . ................ 133

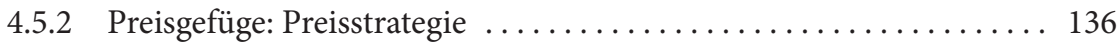

4.5.3 Weiterentwicklung und Nachhaltigkeit: Innovationsstrategie . . . . 142

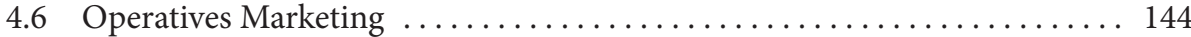

4.7 Portfoliomanagement versus Bauchladen $\ldots \ldots \ldots \ldots \ldots \ldots \ldots \ldots \ldots$ 
4.8 Der Portfoliomanager als interner und externer Vertrieb . . . . . . . . . 150

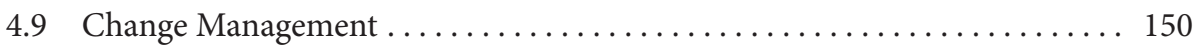

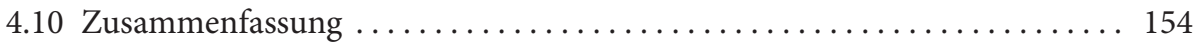

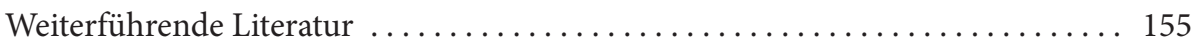

Bücher und Zeitschriften ............................ 155

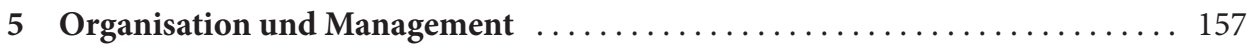

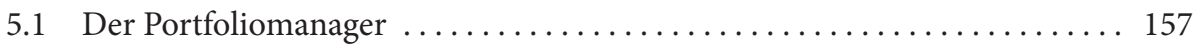

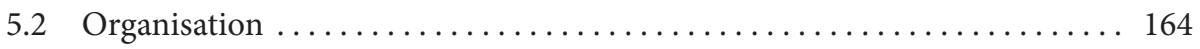

5.2.1 Untergeordnetes Portfoliomanagement ................ 165

5.2.2 Portfoliomanagement als Matrix .................. 168

5.2.3 Portfoliomanagement mit Linienverantwortung . . . . . . . . . . 172

5.2.4 Organisatorische Zuordnung des Portfoliomanagements ........ 174

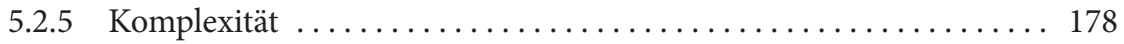

5.3 Natürliche Konflikte im Portfoliomanagement . . . . . . . . . . . . . 182

5.3.1 Widerspruch 1: Produkt versus Linie ................... 184

5.3.2 Widerspruch 2: Produkt versus Projekt ............... 186

5.3.3 Widerspruch 3: Produkt versus Vertrieb ................ 191

5.3.4 Widerspruch 4: Portfolio versus Produkt ................. 195

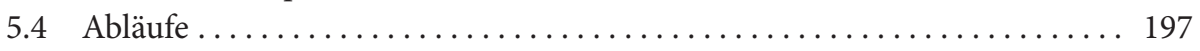

5.4.1 Abstimmung Portfoliomanagement - Vertrieb -

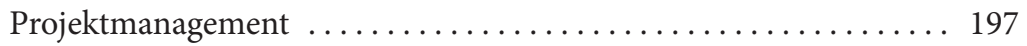

5.4 .2 Information und Kommunikation $\ldots \ldots \ldots \ldots \ldots \ldots \ldots \ldots \ldots \ldots$

5.4 .3 Entscheidungen ............................ 204

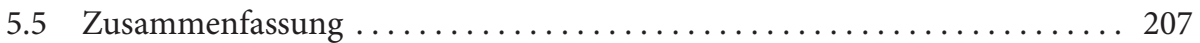

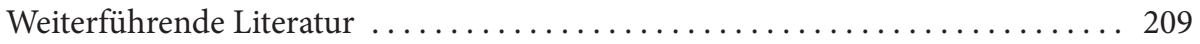

Bücher und Zeitschriften ........................ 209

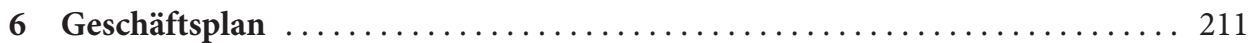

6.1 Erstellung des Geschäftsplanes . . . . . . . . . . . . . . . 213

6.1.1 Die Basis des Geschäftsplanes ist ein solides Geschäftsmodell . . . . 213

6.1.2 Vom Geschäftsmodell zum Geschäftsplan ............... 215

6.2 Inhalte eines Geschäftsplanes . . . . . . . . . . . . . . . 220

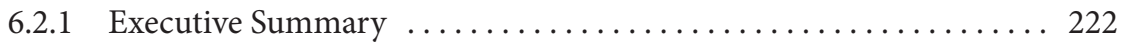

6.2.2 Allgemeine Unternehmensbeschreibung/ Kontext ........... 223

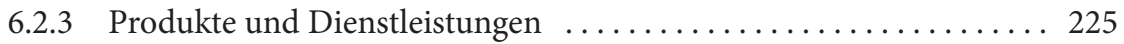

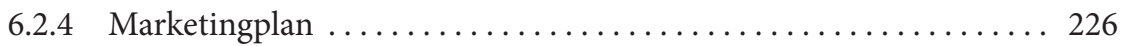

6.2 .5 Umsetzungsplan .......................... 243

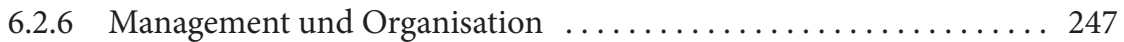

6.2.7 Unternehmensstruktur und Kapitalisierung .............. 250

6.2 .8 Risiken und Chancen . . . . . . . . . . . . . . . . . . . 252

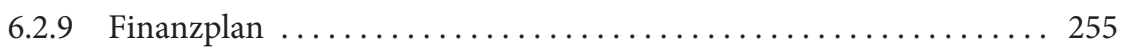

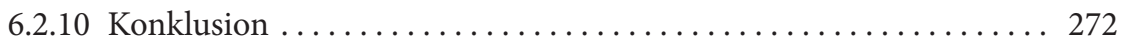




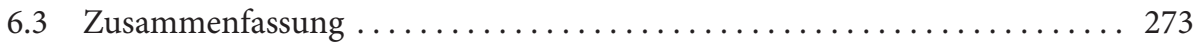

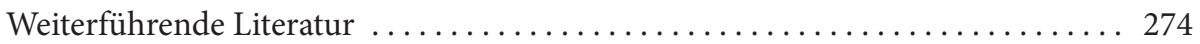

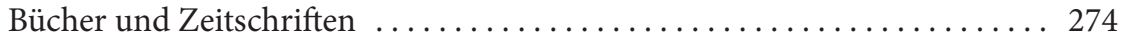

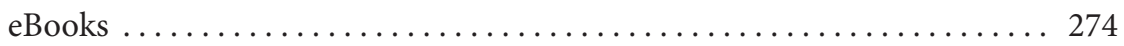

Online-Quellen ................................. 274

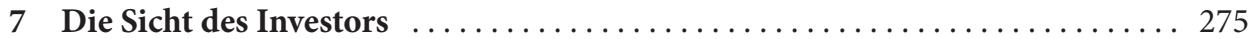

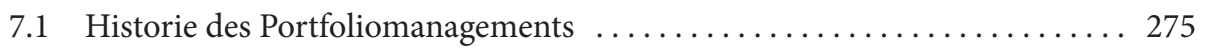

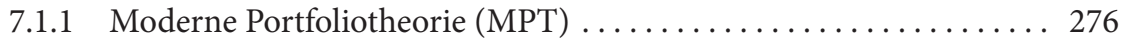

7.1.2 CAPM (Capital Asset Pricing Model) . . . . . . . . . . . . . . . . . . . 279

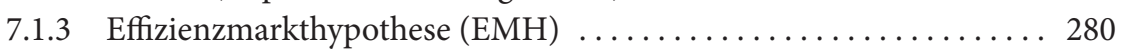

7.1.4 Asset Allocation 1. Generation ...................... 281

7.1.5 Asset Allocation 2. und 3. Generation ................. 287

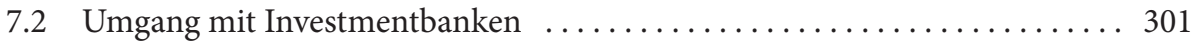

7.2.1 Eine (sehr) kurze Geschichte von Investment-Banking-Services . . 301

7.2.2 Klassische Investment-Banking-Services $\ldots \ldots \ldots \ldots \ldots . \ldots 303$

7.2 .3 Schein und Sein . . . . . . . . . . . . . . . . . . 305

7.3 Gängige Bewertungsmethoden in der Unternehmensanalyse . . . . . . . 312

7.3.1 Discounted Cash Flow/Net Present Value . . . . . . . . . . . . . . . . 313

7.3.2 Comparables/Multiples ...................... 314

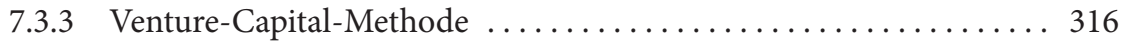

7.3 .4 First-Chicago-Methode . ..................... 318

7.3.5 Methodenwahl ....................................... 320

7.4 Bewertung von Produktportfolios . . . . . . . . . . . . . . 321

7.4.1 Produktneuheiten in Cash-Flow-Annahmen berücksichtigen? ... . . 321

7.4.2 Nützliche Praktikerliste aus Investorensicht ............ 321

7.4.3 Reflexion hinsichtlich Bewertungsmodellen und Verhandlungen ... 322

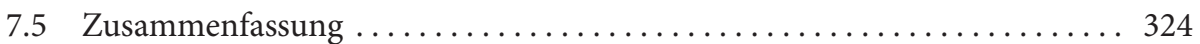

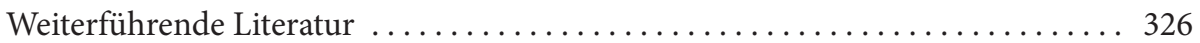

Bücher ..................................... 326

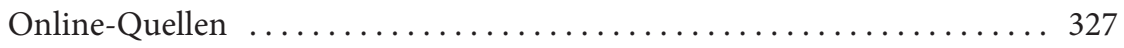

Sachverzeichnis ...................................... 329 


\section{Abbildungsverzeichnis}

Abb. 2.1 Bewertungshorizont: Einzelvorhaben versus Produkt $\ldots \ldots \ldots \ldots \ldots \ldots$

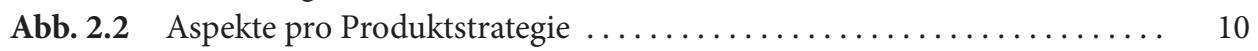

Abb. 2.3 Gesamtentwicklung versus schrittweises Vorgehen .............. 19

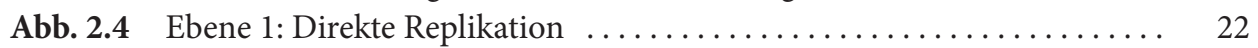

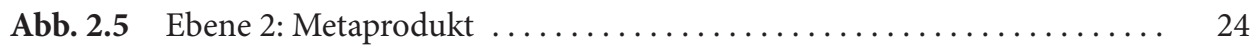

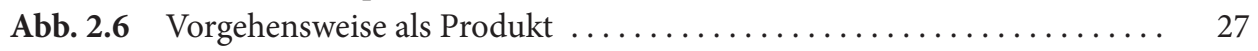

Abb. 2.7 Produktlebenszyklus nach Kotler und Bliemel $\ldots \ldots \ldots \ldots \ldots \ldots \ldots \ldots$

Abb. 2.8 Revidierter Produktlebenszyklus nach Moore . . . . . . . . . . . . . . 37

Abb. 2.9 Portfolio-Sensitivitätsanalyse Schritte $1-3 \ldots \ldots \ldots \ldots \ldots \ldots \ldots \ldots \ldots$

Abb. 2.10 Portfolio-Sensitivitätsanalyse Schritte $4-9 \ldots \ldots \ldots \ldots \ldots \ldots \ldots \ldots \ldots$

Abb. 2.11 Portfolio-Sensitivitätsanalyse Schritte 10 und $11 \ldots \ldots \ldots \ldots \ldots \ldots \ldots$

Abb. 3.1 Abgrenzung des relevanten Marktes $\ldots \ldots \ldots \ldots \ldots \ldots \ldots \ldots \ldots \ldots \ldots$

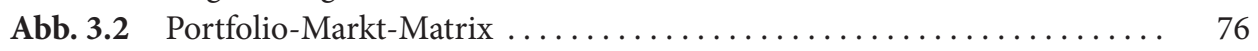

Abb. 3.3 Beispiel einer Produkt-Markt-Matrix $\ldots \ldots \ldots \ldots \ldots \ldots \ldots \ldots \ldots \ldots$

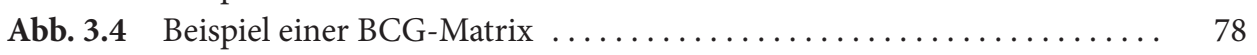

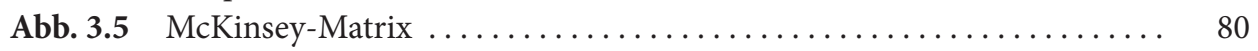

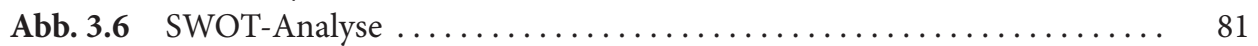

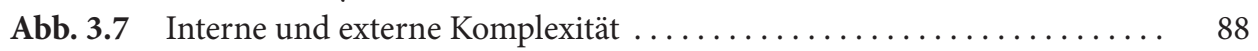

Abb. 3.8 Anstieg der Komplexität bei Produkten aufgrund wachsender

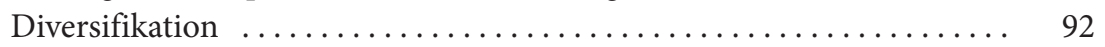

Abb. 3.9 Phasen des Komplexitätsmanagements .................... 95

Abb. 4.1 Der Prozess des strategischen Marketings $\ldots \ldots \ldots \ldots \ldots \ldots \ldots \ldots \ldots . \ldots \ldots$

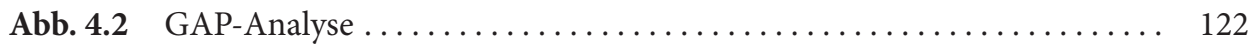

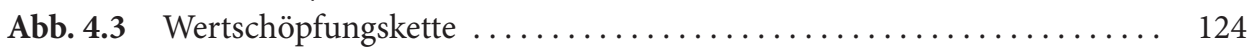

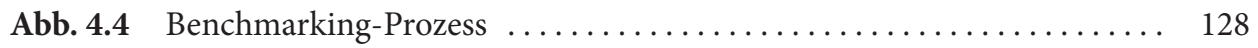

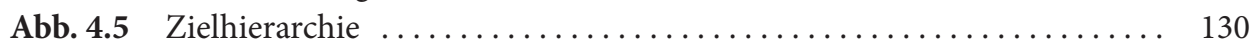

Abb. 4.6 Säulenmodell einer Portfoliostrategie .................... 134

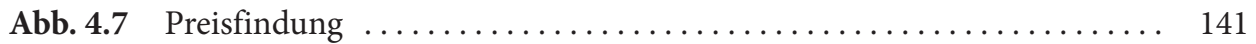

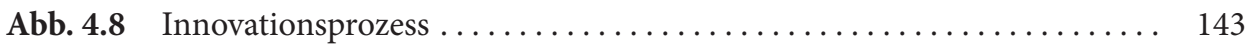

Abb. 4.9 Innovationsprozess: Einflussbereiche des Portfoliomanagers . . . . . . . 144 
Abb. 4.10 Definition des Marketingmix . . . . . . . . . . . . . . . . . 146

Abb. 5.1 Produktmanager als Querschnittsfunktion $\ldots \ldots \ldots \ldots \ldots \ldots \ldots \ldots . \ldots 159$

Abb. 5.2 Untergeordnetes Portfoliomanagement . ................... 167

Abb. 5.3 Portfoliomanagement als Matrix $\ldots \ldots \ldots \ldots \ldots \ldots \ldots \ldots \ldots \ldots \ldots$

Abb. 5.4 Portfoliomanagement mit Linienverantwor-tung $\ldots \ldots \ldots \ldots \ldots \ldots \ldots \quad 172$

Abb. 5.5 Produkt- und Portfoliomanagement als Matrix . . . . . . . . . . . . 177

Abb. 5.6 Produkt- und Portfoliomanagement mit direkter Berichtslinie . . . . . 177

Abb. 5.7 Komplexitätsverarbeitungskapazität einer Organisation . . . . . . . . . 179

Abb. 5.8 Optimierung durch Erhöhung der Binnenkomplexität . . . . . . . . . 179

Abb. 5.9 Beispiel Anlagenbau: Lernkurve bei neuem Portfolioelement . . . . . . . . 201

Abb. 6.1 Wichtige Einflussfaktoren bei Überlegungen rund um Portfolioelemente ................................... 214

Abb. 6.2 Erfolgskritische Meilensteine bei der Erstellung von Geschäftsplänen . . . 218

Abb. 6.3 Beispiele für Abhängigkeiten zwischen Portfolioelementen . . . . . . . . 218

Abb. 6.4 Wirksamkeit in Abhängigkeit von der Größe des Zielmarktes . . . . . . . 230

Abb. 6.5 Alternative Distributionsstrategien ................... 234

Abb. 6.6 Direkte und indirekte Kosten für Portfolioelemente ............. 258

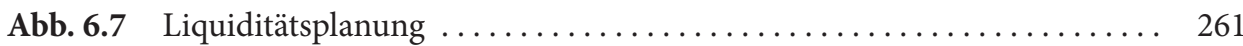

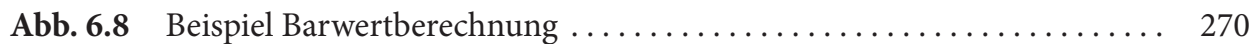

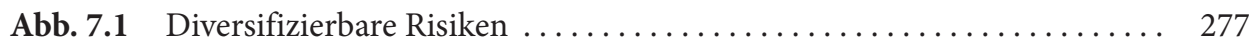

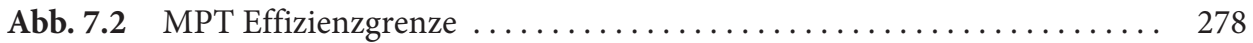

Abb. 7.3 Säulenheilige der ersten Generation von Asset-Allocation-Modellen . . 281

Abb. 7.4 Asset-Allocation-Generationen ....................... 282

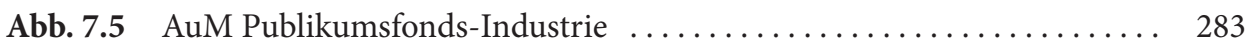

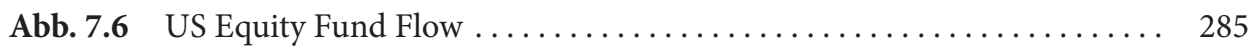

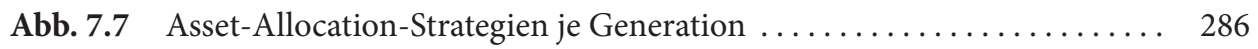

Abb. 7.8 Diversifikation des Yale-Portfolios Mitte der 1990er Jahre . . . . . . . . 290

Abb. 7.9 UOBAM Investment Clock . . . . . . . . . . . . . . . . . . . . . . . . 293

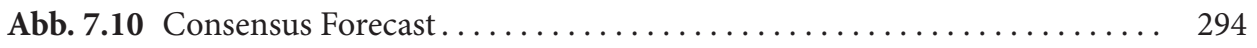

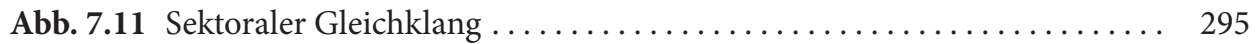

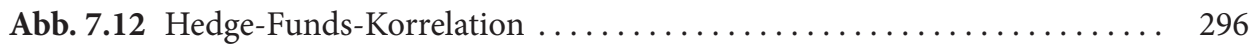

Abb. 7.13 Asset Class Allocation . . . . . . . . . . . . . . . . . . . . . . . . . 296

Abb. 7.14 Risk Allocation ................................. 297

Abb. 7.15 Asset-Allocation-Modell-Historie . . . . . . . . . . . . . . . . . . 300

Abb. 7.16 IB Service-Tree .................................. 303

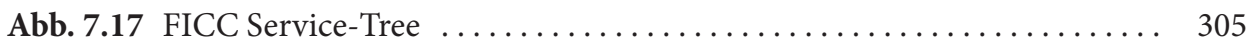

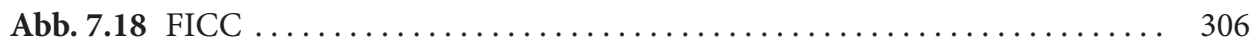

Abb. 7.19 Karriereverlauf Analyst ....................... 307

Abb. 7.20 Forecast Korrekturen . . . . . . . . . . . . . . . . . . . . . . . . . . . . . . 309

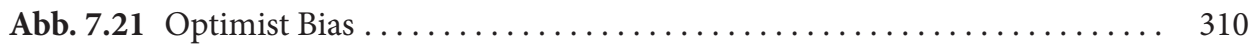

Abb. 7.22 Optimist Bias .............................. 310 


\section{Tabellenverzeichnis}

Tab. 3.1 Einstufung von Datenmaterial im Rahmen der Marktforschung . . . . . . 72

Tab. 3.2 Arten und Eigenschaften von USPs $\ldots \ldots \ldots \ldots \ldots \ldots \ldots \ldots \ldots \ldots \ldots$

Tab. 4.1 Beispiel eines Fragebogens zur unternehmensinternen Bewertung ...... 118

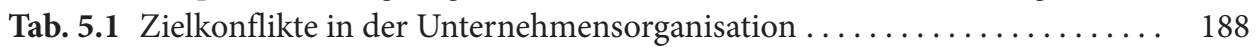

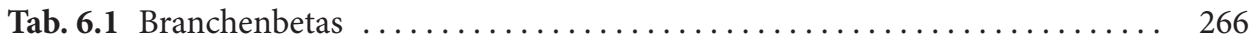

Tab. 6.2 Zinssätze nach Risikokategorien ...................... 269

Tab. 7.1 Asset Allocation bei steigender Illiquidität $\ldots \ldots \ldots \ldots \ldots \ldots \ldots \ldots \ldots . \ldots \ldots$

Tab. 7.2 Asset Allocation von Asset Manager je Assetklasse . . . . . . . . . . . . . 292

Tab. 7.3 Asset Allocation von Pensionsfonds je Assetklasse .............. 292

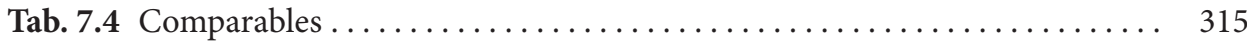




\section{Die Autoren}

Dr. Reinhard Grimm MBA arbeitet als Geschäftsbereichsleiter in einem international erfolgreichen Hochtechnologie-Konzern in Wien und lehrt an universitären sowie außeruniversitären Einrichtungen. Die inhaltlichen Schwerpunkte seiner beruflichen Tätigkeit sind vor allem im Kontext der geschäftlichen Verantwortung für das Produktportfolio von Unternehmensbereichen und im Aufbau neuer Geschäftsfelder angesiedelt. In diesem Zusammenhang leitete er auch mehrere Programme zur Organisationsentwicklung und Reorganisation von Abteilungen in Großunternehmen.

Neben einem Technikstudium sind seine wissenschaftlichen Schwerpunkte vor allem Projektmanagement, soziale Kompetenz und Gruppendynamik. Zu diesem Themenkreis verfasste Reinhard Grimm bereits mehrere Bücher und Artikel.

Mag. Markus Schuller MBA, MScFE Markus Schuller ist Gründer von Panthera Solutions, einem Anbieter von Strategic Asset Allocation Intelligence mit Sitz im Fürstentum Monaco, sowie Adjunct Professor an der International University of Monaco.

Er blickt auf über 15 Jahre Erfahrung im Handeln, Strukturieren und Managen von traditionellen und alternativen Investmentprodukten zurück. Mit Panthera Solutions bietet er professionellen Investoren eine Überleitung in die dritte Generation von Asset-Allocation-Optimierungstechniken an. Markus Schuller spricht regelmäßig auf internationalen Investment-Konferenzen zum Thema Asset Allocation und kommentiert den Finanzmarkt für deutschsprachige Qualitätsmedien.

Raimund Wilhelmer unterstützt mittelständische IT-Unternehmen bei der geschäftlichen Entwicklung von Innovationen und deren Etablierung am Markt.

In seiner mehr als 20-jährigen beruflichen Tätigkeit hat er umfassende Erfahrung im Produktmanagement und Portfoliomanagement in Deutschland, Österreich sowie der Schweiz gesammelt und in operativer wie beratender Funktion mehreren Start-ups mit innovativen Geschäftsmodellen zum Erfolg verholfen. In seiner bisherigen Laufbahn kon- 
zentrierte er sich vor allem auf die operative Umsetzung des Prozesses von der Idee über die Entwicklung einer Go-to-Market-Strategie bis hin zur praktischen Realisierung des Geschäftsmodells.

Zudem ist er an zahlreichen nationalen und internationalen Initiativen, die den Aufbau und die wirtschaftliche Entfaltung von Unternehmen fördern, aktiv beteiligt. 